\title{
Quantitative Analysis of Photodynamic Therapy Effects in Rat Mammary Tumor Vascular Density Using Image-Pro plus Software
}

\author{
Isabelle Ferreira $^{1^{*}}$, Camilo Bulla ${ }^{2}$, Wes Baumgartner ${ }^{2}$, Vanderlei Salvador Bagnato ${ }^{3}$, \\ Noeme Sousa Rocha ${ }^{1}$ \\ ${ }^{1}$ Veterinary Clinics Department, School of Veterinary Medicine and Animal Science, \\ Universidade Estadual Paulista, Botucatu, Brazil \\ ${ }^{2}$ Department of Pathobiology and Population Medicine, College of Veterinary Medicine, \\ Mississippi State University, Starkville, USA \\ ${ }^{3}$ Department of Physics and Science of the Materials, São Carlos Physics Institute, \\ University of São Paulo (USP), São Carlos, Brazil \\ Email: *iferreira_16@hotmail.com
}

Received June 9, 2013; revised July 9, 2013; accepted August 9, 2013

Copyright (c) 2013 Isabelle Ferreira et al. This is an open access article distributed under the Creative Commons Attribution License, which permits unrestricted use, distribution, and reproduction in any medium, provided the original work is properly cited.

\begin{abstract}
Photodynamic therapy (PDT) is a treatment modality that has advanced rapidly in recent years. It causes tissue and vascular damage with the interaction of a photosensitizing agent (PS), light of a proper wavelength, and molecular oxygen. Evaluation of vessel damage usually relies on histopathology evaluation. Results are often qualitative or at best semi-quantitative based on a subjective system. The aim of this study was to evaluate, using CD31 immunohistochemistry and image analysis software, the vascular damage after PDT in a well-established rodent model of chemically induced mammary tumor. Fourteen Sprague-Dawley rats received a single dose of 7,12-dimethylbenz(a)anthraxcene (80 $\mathrm{mg} / \mathrm{kg}$ by gavage), treatment efficacy was evaluated by comparing the vascular density of tumors after treatment with Photogem $^{\otimes}$ as a PS, intraperitoneally, followed by interstitial fiber optic lighting, from a diode laser, at $200 \mathrm{~mW} / \mathrm{cm}$ and light dose of $100 \mathrm{~J} / \mathrm{cm}$ directed against his tumor (7 animals), with a control group (6 animals, no PDT). The animals were euthanized 30 hours after the lighting and mammary tumors were removed and samples from each lesion were formalin-fixed. Immunostained blood vessels were quantified by Image Pro-Plus version 7.0. The control group had an average of $3368.6 \pm 4027.1$ pixels per picture and the treated group had an average of $779 \pm 1242.6$ pixels per area $(\mathrm{P}<$ 0.01 ), indicating that PDT caused a significant decrease in vascular density of mammary tumors. The combined immunohistochemistry using CD31, with selection of representative areas by a trained pathology, followed by quantification of staining using Image Pro-Plus version 7.0 system was a practical and robust methodology for vessel damage evaluation, which probably could be used to assess other antiangiogenic treatments.
\end{abstract}

Keywords: Mammary Tumors; Photodynamic Therapy; Vascular Damage

\section{Introduction}

Photodynamic therapy (PDT) is a treatment modality with uses in oncology and cardiovascular disease [1]. The clinical treatment regimen consists of induction of cytotoxicity in proliferative cells through the interaction of a photosensitizing agent (PS), light of a proper wavelength, and molecular oxygen. Vascular and direct cell damages contribute to tumor destruction. The vessels are an important target for PDT using many photosensitizers [2]. With a derivative of hematoporphyrin as PS and

\footnotetext{
${ }^{*}$ Corresponding author.
}

large interval between the administration of PS and the lighting of the lesion, the action of PDT is more cellular, however if you use a shorter time interval the vascular response prevails [3].

Evaluation of vessel damage after antiangiogenic treatment in in vivo models usually relies on histopathology evaluation by a trained pathologist, which can be subjective. Results are often qualitative or at best semiquantitative based on a subjective system. CD31 is a transmembrane glycoprotein of the immunoglobin superfamily that plays an important role in cell-cell adhesion, it's involved in angiogenesis, and it is a well-established marker for 
endothelial cells. The aim of this study was to evaluate, using CD31 immunohistochemistry and image analysis software, the vascular damage after PDT in a well-established rodent model of chemically induced mammary tumor.

\section{Material and Methods}

\subsection{Animals}

Thirteen young, nulliparous, Sprague-Dawley female rats were used in this study. The animals were fed with appropriate rat food in pellets, given filtered water, and kept under ideal conditions of temperature, humidity and light.

\subsection{Photosensitizer}

Photogem (Photogem, Moscow, Russia), a hematoporphyrin derivative complex, was used as PS. A stock solution of $5 \mathrm{mg} / \mathrm{mL}$ in $20 \mathrm{mM}$ phosphate-buffered solution with $0.9 \% \mathrm{NaCl}, \mathrm{pH} 7.4$ (PBS) was stored in the dark at $4^{\circ} \mathrm{C}$.

\subsection{Light Source}

The light source used was the laser diode Ceralas $630^{\circledR}$ (Ceramoptec, Germany), which produces up to $2 \mathrm{~W}$ of optical power at a wavelength of $630 \mathrm{~nm}$. The intensity applied was $200 \mathrm{~mW} / \mathrm{cm}$.

\subsection{Experimental Design}

Mammary tumors were induced by a single dose of 80 $\mathrm{mg} / \mathrm{kg}$ of 7,12-dimethylbenz(a)anthracene (DMBA, SigmaAldrich, St. Louis, MO) diluted in soybean oil given by gavage. After induction, the animals underwent daily viability inspection, and weekly physical examination, until tumor development.

After that, the animals were divided into two groups: control group (6 animals, no PDT); and the treated group with Photogem ${ }^{\circledR}$ intraperitoneally, followed by interstitial fiber optic lighting 24 hours later, from a diode laser, at $200 \mathrm{~mW} / \mathrm{cm}$ and light dose of $100 \mathrm{~J} / \mathrm{cm}$ directed against the tumor (7 animals). For this procedure, the animals were anesthetized with intramuscular injectioof xylazine $2 \%$ (20 mg) and ketamine (50 mg) at the dosage of 0.2 $\mathrm{ml}$ per $100 \mathrm{~g}$ of rat.

Thirty hours after the PDT treatment, the animals were deeply anesthetized with isoflurane and then euthanized by transaction of the jugular veins. The lesions were excised and analyzed by routine hematoxylin and eosin and immunohistochemistry.

\subsection{Immunohistochemistry}

The slides were placed for 30 minutes in the oven at $65^{\circ} \mathrm{C}$ after incubation in xylene (30 minutes), dipped in $100 \%$ ethanol, then in $70 \%$ ethanol and rinsed in distilled water. Antigen retrieval was performed using Antigen Retrieval Buffer (Dako, Carpinteria, CA) in steamer for 30 minutes. After this procedure, the slides were rinsed with distilled water. Blood vessels were identified using a rabbit polyclonal antibody against CD31 (PECAM-1, Biorbyt, San Francisco, CA). The detection system used was LSAB2 System-HRP for rat specimens (Dako, Carpinteria, CA). The slides were stained with the reagent 3,3'diaminobenzidine and counterstained with hematoxylin and eosin.

\subsection{Image Analysis}

Image Pro-Plus version 7.0 (Media Cybernetics, Rockville, MD) system was chosen for the image analysis because it allows a high degree of replication and automation of image-processing steps and it is one of the most popular systems used currently by investigators. Pictures from three different areas of lowest vascular density of each tumor were submitted to the software for analysis. The colors correspondent to positive staining were recorded and numbers of pixels containing those colors per area were automatic counted on all pictures by the software, and interpreted as the vascular density of the lesion.

\subsection{Statistical Analysis}

To compare the vascular density in the two groups, statistical analysis of the number of pixels correspondent to CD31 staining was done by Student's t-test, using SAS version 9.2 (SAS Institute Inc, Cary, NC, USA). A value of $\mathrm{P}<0.05$ was considered to be statistically significant.

\section{Results and Discussion}

The analysis showed that the control group had an average of $3368.6 \pm 4027.1$ pixels per picture (Figure 1) and the treated group had an average of $779 \pm 1242.6$ pixels (Figure 2) $(\mathrm{P}<0.01)$, indicating that the PDT treatment induced a significant vascular damage.

Angiogenesis has been long recognized as a key step in tumor growth and metastasis development [4]. By secreting various growth factors, tumors induce blood vessel growth (angiogenesis), which allows tumor expansion [5]. Thus, angiogenesis is a necessary and required step for transition from a small harmless cluster of cells to a large tumor and is also required for the spread of a tumor, invasion and/or metastasis. To date, it has been demonstrated by innumerous works that tumors unable to successful induce angiogenesis will remain microscopic. The inhibition of angiogenesis is an attractive therapeutic approach to control tumor progression [6]. PDT mechanism of action is the local generation of cytotoxic singlet 


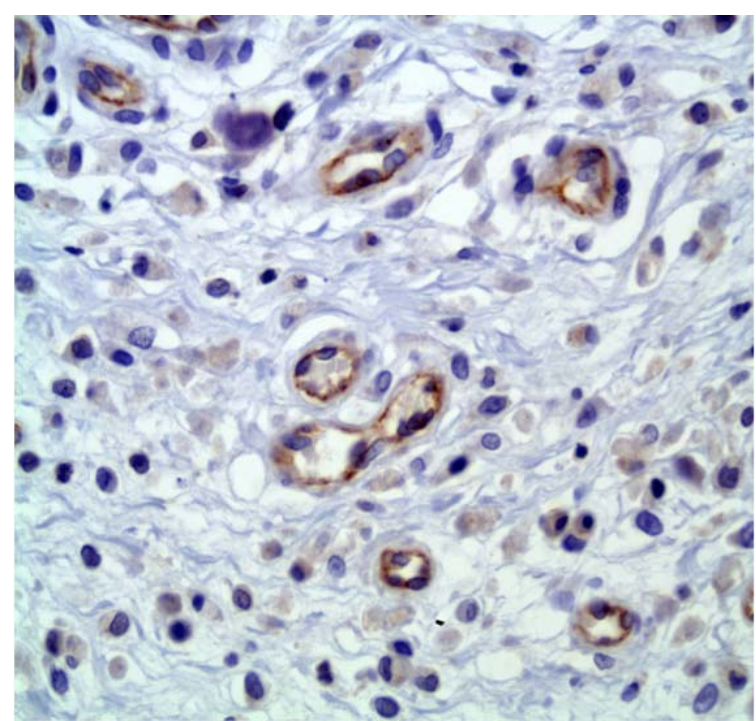

Figure 1. CD31 immunostaining of control group. $50 \times \mathrm{im}-$ mersion objective.

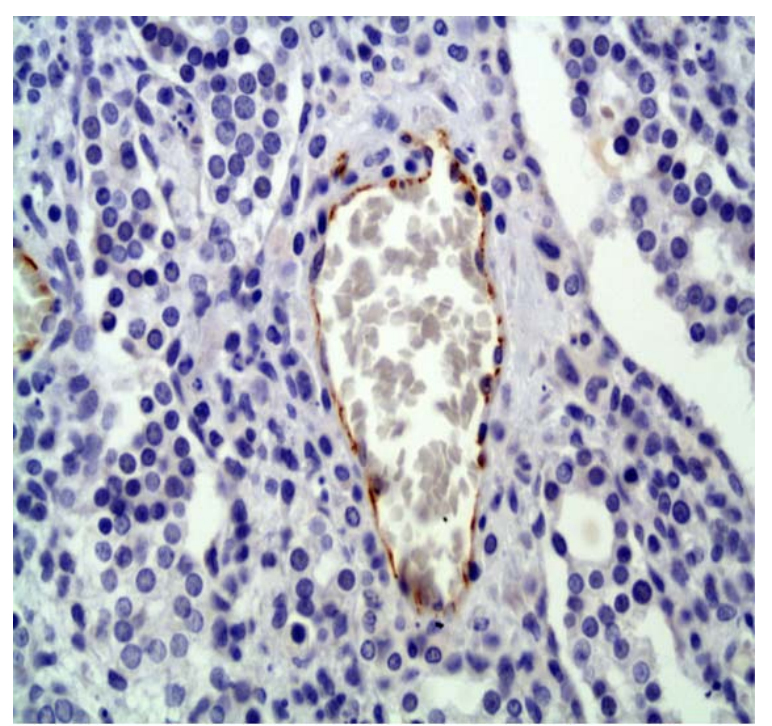

Figure 2. CD31 immunostaining of treated group. $50 \times \mathrm{im}-$ mersion objective.

oxygen $\left({ }^{1} \mathrm{O} 2\right)$, which results in damage to tumor cells and stroma, especially the tumor vasculature, and usually is accompanied by an inflammatory response [7]. It was demonstrated that tumors treated with short drug light interval (DLI) PDT exhibited greater tumor regression compared to long DLI PDT, and that was attributed to a greater vascular damage in the tumor [8].

Microvessel density evaluation can be done in several different ways. Histopathology only is the most subjective and prone to errors. Immunohistochemistry using antibodies against markers of vessels allows for more precise quantification of microvessels than by histology alone, but still relies on trained pathologists for interpretation and thus can be subjective. Available markers in- clude vWF, CD34, VEGF, and CD31. The latter is the most sensitive and specific for endothelial cells in paraffin sections [9-12]. The combined use of immunohistochemistry with selection of representative areas by a trained pathologist, and further quantification of positivity by a software analysis may address some of the subjectivity of the other methods.

In the present study, we demonstrated a significant decrease in microvascular density induced by PDT in rat mammary tumor. Our results corroborate with several others studies that used PDT against different tumor models. For example, researchers found a significant decrease in intensity of CD31-stained blood vessels in murine colon carcinoma cells samples in PDT treated mice compared to non-treated controls [7]. Another study also showed evidence of vascular damage after PDT by CD31 staining, in mice bearing colon-26 tumors. It was demonstrated that low dose PDT induces changes in vessel permeability. However, these changes were a subtle injury that was below the threshold needed to achieve tumor destruction. But with an increase of dosage, the treatment stimulates a catastrophic vascular collapse and dissolution, leading to tumor death [13]. Bhuvaneswari and collaborators [8] treated at a human bladder carcinoma xenograft model with long and short DLI PDT. At 30 days after the treatment, they observed that although the short DLI PDT effect on the tumor had diminished and minimal staining was observed, comparably higher CD31-stained blood vessels were noticed in long DLI PDT, suggesting neovessel formation and tumor regrowth. Interestingly, they also observed upregulation of proangiogenic genes after PDT, which indicates a greater need for combination therapy by including antiangiogenic agents that target specific pathways.

\section{Conclusion}

Evaluation of the images shows that PDT causes a significant decrease in vascular density of mammary tumors, what is one of the advantages of this modality of treatment. The combined immunohistochemistry using CD31, with selection of representative areas by a trained pathology, followed by quantification of CD31 staining using Image Pro-Plus version 7.0 system was a practical and robust methodology for vessel damage evaluation, that probably could be used to assess other antiangiogenic treatments.

\section{Acknowledgements}

The authors are greatly indebted to FAPESP (Fundação de Amparo a Pesquisa do Estado de São Paulo) for Financial Support, CePOF-IFSCUSP (Optics and Photonics Research Center, Instituto de Física de São Carlos, Universidade de São Paulo), and Sandra Curotto Bulla 
for valuable help with manuscript revision.

\section{REFERENCES}

[1] V. H. Fingar, P. K. Kik, P. S. Haydon, P. B. Cerrito, M. Tseng, E. Abang and T. J. Wieman, "Analysis of Acute Vascular Damage after Photodynamic Therapy Using Benzoporphyrin Derivative (BPD)," British Journal of Cancer, Vol. 79, No. 11-12, 1999, pp. 1702-1708. doi:10.1038/sj.bjc.6690271

[2] V. H. Fingar, T. J. Wieman, S. A. Wiehle and P. B. Cerrito, "The Role of Microvascular Damage in Photodynamic Therapy: The Effect of Treatment on Vessel Constriction, Permeability, and Leukocyte Adhesion,” Cancer Research, Vol. 52, 1992, p. 4914.

[3] L. B. Li and R. C. Luo, "Effect of Drug-Light Interval on the Mode of Action of Photofrin Photodynamic Therapy in a Mouse Tumor Model," Lasers in Medical Science, Vol. 24, No. 4, 2009, pp. 597-603. doi:10.1007/s10103-008-0620-9

[4] J. Folkman, "Angiogenesis in Cancer, Vascular, Rheumatoid and Other Disease," Nature Medicine, Vol. 1, No. 1, 1995, pp. 27-31. doi:10.1038/nm0195-27

[5] D. L. Nielsen, M. Andersson, J. L. Andersen and C. Kamby, "Antiangiogenic Therapy for Breast Cancer," Breast Cancer Research, Vol. 12, No. 5, 2010, p. 209. doi:10.1186/bcr2642

[6] J. Harper and M. A. Moses, "Molecular Regulation of Tumor Angiogenesis: Mechanisms and Therapeutic Implications,” EXS, Vol. 96, 2006, pp. 223-268.

[7] B. W. Henderson, S. O. Gollnick, J. W. Snyder, et al., "Choice of Oxygen-Conserving Treatment Regimen Determines the Inflammatory Response and Outcome of Photodynamic Therapy of Tumors," Cancer Research, Vol. 64, 2004, pp. 2120-2126. doi:10.1158/0008-5472.CAN-03-3513

[8] R. Bhuvaneswari, Y. Y. Gan, S. S. Lucky, W. W. L. Chin, S. M. Ali, K. C. Soo and M. Olivo, "Molecular Profiling of Angiogenesis in Hypericin Mediated Photodynamic Therapy,” Molecular Cancer, Vol. 7, No. 1, 2008, p. 56. doi:10.1186/1476-4598-7-56

[9] A. Bottini, A. Berruti, A. Bersiga, M. P. Brizzi, G. Allevi, G. Bolsi, S. Aguggini, A. Brunelli, E. Betri, D. Generali, L. Scaratti, G. Bertoli, P. Alquati and L. Dogliotti, "Changes in Microvessel Density as Assessed by CD34 Antibodies after Primary Chemotherapy in Human Breast Cancer,” Clinical Cancer Research, Vol. 8, No. 6, 2002, pp. 1816-1821.

[10] D. C. Chhieng, S. O. Tabbara, E. F. Marley, L. I. Talley and A. R. Fros, "Microvessel Density and Vascular Endothelial Growth Factor Expression in Infiltrating Lobular Mammary Carcinoma,” Breast Journal, Vol. 9, No 3, 2003, pp. 200-207. doi:10.1046/j.1524-4741.2003.09311.x

[11] D. Wang, C. R. Stockard, L. Harkins, P. Lott, C. Salih, K. Yuan, D. Buchsbaum, A. Hashim, M. Zayzafoon, R. Hardy, O. Hameed, W. Grizzle and G. P. Siegal, "Immunohistochemistry for the Evaluation of Angiogenesis in Tumor Xenografts,” Biotechnic \& Histochemistry, Vol. 83, No. 3, 2008, pp. 179-189. doi:10.1080/10520290802451085

[12] P. Mahzouni, F. Mohammadizadeh, K. Mougouei, N. A. Moghaddam, A. Chehrei and A. Mesbah, "Determining the Relationship between 'Microvessel Density' and Different Grades of Astrocytoma Based on Immunohistochemistry for 'Factor VIII-Related Antigen' (von Willebrand Factor) Expression in Tumor Microvessels," Indian Journal of Pathology and Microbiology, Vol. 53, No. 4, 2010, pp. 605-610. doi:10.4103/0377-4929.71996

[13] M. Seshadri, J. A. Spernyak, R. Mazurchuk, S. H. Camacho, A. R. Oseroff, R. T. Cheney and D. A. Bellnie, “Tumor Vascular Response to Photodynamic Therapy and the Antivascular Agent 5,6-Dimethylxanthenone-4-Acetic Acid: Implications for Combination Therapy," Clinical Cancer Research, Vol. 11, 2005, p. 4241. doi:10.1158/1078-0432.CCR-04-2703 